SUNY - NTG - $95-13$

\title{
The Constituent Quark Limit and the Skyrmion Limit of Chiral Quark Soliton Model
}

\author{
Michał Praszałowicz ${ }^{(1) *}$, Andree Blotz $^{(2,3)} \dagger$ and Klaus Goeke ${ }^{(3)} \ddagger$ \\ (1) Institute of Physics, Jagellonian University, Reymonta 4, \\ 30-059 Kraków, Poland \\ (2) Department of Physics, State University of New York at Stony Brook, \\ Stony Brook, New York, 11794, USA \\ (3) Institute for Theoretical Physics II, \\ P.O. Box 102148, Ruhr-University Bochum, \\ D-W-44780 Bochum, Germany
}

(July 27, 2021)

\begin{abstract}
We calculate $g_{\mathrm{A}}^{(3)}$ and $g_{\mathrm{A}}^{(0)}$ in the Chiral Quark-Soliton Model (or equivalently in semibosonized Nambu-Jona-Lasinio model) in the limit of small and large soliton size. In small soliton limit we recover the results of the Constituent Quark Model. The agreement between the two models is achieved due to the recently calculated $1 / N_{\mathrm{c}}$ contributions to $g_{\mathrm{A}}^{(3)}$ and to $g_{\mathrm{A}}^{(0)}$. In the case of $g_{\mathrm{A}}^{(3)}$ these terms arise from the time-ordering of the collective operators. For large solitons simple scaling arguments show that the $1 / N_{\mathrm{c}}$ contributions vanish with the inverse power of the soliton size.
\end{abstract}

\footnotetext{
*supported by Alexander von Humboldt Foundation email: michal@thrisc.if.uj.edu.pl

${ }^{\dagger}$ supported by Alexander von Humboldt Foundation email: andreeb@luigi.physics.sunysb.edu

‡email: goeke@hadron.tp2.ruhr-uni-bochum.de
} 
The Constituent Quark Model (CQM), although often considered as naive, has been often used to calculate a priori dynamical hadronic matrix elements in terms of simple group-theoretical factors. The most prominent prediction of the CQM, namely the value of the singlet axial constant $g_{\mathrm{A}}^{(0)}=1$, caused the so-called "spin crisis". The CQM prediction [1] for $g_{\mathrm{A}}^{(3)}=\left(N_{\mathrm{c}}+2\right) / 3$, which overshoots the experimental value of 1.26 , triggered the interest in the possible renormalization of a quark axial coupling away from 1 [2, 3]. On the other extreme Skyrme-like models, in which baryons are considered as nontrivial configurations of the classical pion-sigma chiral fields, gave numbers in flat contradiction with the CQM (and experiment): $g_{\mathrm{A}}^{(3)}<1$ [4] and $g_{\mathrm{A}}^{(0)}=0$ [5]. These models are subjected to a semiclassical quantization of the rotational zero-modes - only then the baryon spectrum is reproduced [4]. The class of interpolating models, like the quark Nambu-Jona-Lasinio (NJL) model (or equivalently Chiral Quark-Soliton Model) [6-11], in which both quarks and chiral fields are present, seemingly did not improve upon the $g_{\mathrm{A}}$ 's, being on the low side in rough agreement with the Skyrme model. However not only the values of the $g_{\mathrm{A}}$ 's have been wrong, but also the $N_{\mathrm{c}}$ counting. In the NJL model and in the Skyrme model as well, $g_{\mathrm{A}}^{(3)}$ scales like $N_{\mathrm{c}}$, however the $\mathcal{O}(1)$ terms, which in the CQM are of utmost importance, seemed to vanish identically. The same concerns the $\mathcal{O}(1)$ contributions to $g_{\mathrm{A}}^{(0)}$ which in the CQM sacles like 1.

Recently $\mathcal{O}(1)$ corrections to the $g_{\mathrm{A}}$ 's and to the magnetic moments have been calculated in the NJL model [12 [15] and also in the chiral bag model [16]. Not only have they significantly improved the numerics of the NJL model, but also the $N_{\mathrm{c}}$ scaling, as predicted by the CQM, has been recovered. On the contrary in the Skyrme-like models based on a local effective meson lagrangian these corrections vanish identically Ref. [17].

The origin of these - for a long time overlooked - corrections (as has been shown in detail in Refs. [13 [15]) is twofold : 1) the NJL model is formulated in terms of a non- 
local effective action, namely in terms of the fermion determinant, and the operators appearing in the path integral for matrix elements have to be time-ordered, 2) the seemingly commuting c-number quantities like angular velocities and the Euler angles in fact do not commute, since upon the semiclassical quantization they are promoted to quantum operators. Apart from these time-ordered corrections there are also terms which come from the anomalous (or imaginary) part of the effective action (as in the case of $\left.g_{\mathrm{A}}^{(0)}\right)$. In this order of the $1 / N_{\mathrm{c}}$ expansion their emergence does not depend on the time order of the collective operators.

In this short note we show, that in the small soliton limit of the NJL model the results of the CQM for the $g_{\mathrm{A}}$ 's are recovered indepently on the model parameters. Indeed, when the soliton ceases to exist the quarks, which are normally bound in a potential created by the chiral fields, reach the plane wave orbits of the free Dirac particles [9]. Moreover, the contribution of the Dirac sea (or equivalently of the chiral fields) vanishes in this limit. Strictly speaking the Constituent Quark Limit can be accomplished by tuning the constituent quark mass $m \rightarrow 0$, in such a way that for each $m$ the selfconsistent soliton solution is obtained. Since for some critical $m_{\mathrm{cr}}$ the bound state solution ceases to exist even if the soliton has finite size, we choose first to push $r_{0} \rightarrow 0$ artificially for some unspecified $m>m_{\mathrm{cr}}$, for which the selfconsistent soliton solution does exist. It is then enough to observe, that the result does not depend on $m$.

Furthermore, on the basis of a simple scaling argument we show, that in the large soliton limit the new corrections die out. Therefore in this limit the predictions of local mesonic effective theories are parametrically reproduced. The picture which emerges is therefore very appealing: the small and large soliton limits of the NJL model coincide with the previously obtained results within the CQM and the Skyrme model. Were the new non-local $\mathcal{O}(1)$ corrections equal to zero, the nice correspondence with the CQM 
would be completely lost.

Let us first briefly remind the philosophy and main ingredients of the NJL model. Our starting point is a semibosonized Euclidean action of the NJL model [13,15]:

$$
S_{\text {eff }}[U]=-\operatorname{Sp} \log \left\{-i \not \partial+m U^{\gamma_{5}}\right\}
$$

where $\gamma$ matrices are antihermitian, the $\mathrm{SU}(2)$ matrix $U^{\gamma_{5}}=\exp \left(i \gamma_{5} \vec{\tau} \vec{\pi} / F_{\pi}\right)$ describes chiral fields and $m$ is the constituent quark mass. Eq.(1) has to be regularized. For the hedgehog Ansatz $U_{0}^{\gamma_{5}}=\cos P(r)+i \vec{n} \vec{\tau} \gamma_{5} \sin P(r)$ with the profile function satisfying the boundary conditions $P(0)=\pi$ and $P(\infty)=0$, the model has solitonic solutions. The existence of the soliton is achieved due to the interplay between the valence level and the energy of the continuum states. All quantities have therefore typically two contributions which we subsequently call valence and sea respectively.

If the soliton size $r_{0}$ (defined through $P=P\left(r / r_{0}\right)$ ) decreases, the valence level joins the upper continuum and the sea contribution vanishes. For large solitons the valence level sinks into the Dirac sea and the explicit contribution of the valence level disappears. In this way the model interpolates between the CQM and the Skyrme-like models 日.

The energy of the soliton is given as the regularized sum of the eigenenergies of the Dirac hamiltonian:

$$
H\left(U_{0}\right)=-i \gamma_{4}\left(-i \gamma_{k} \partial_{k}+m U_{0}^{\gamma_{5}}\right)
$$

H commutes neither with the isospin $(T)$, the spin $(S)$, nor with the angular momentum $(L)$, but only with the so called grand-spin $G=T+J=T+L+S$.

\footnotetext{
${ }^{1}$ One should stress, however, that the four-derivative term from the NJL model does not coincide with the one of the Skyrme model. It is cutoff sensitive and it can stabilize the soliton only for very large constituent masses [10.
} 
To appreciate the way the constituent quark limit of the NJL model is actually reached, requires a careful analysis of the wave functions of the hamiltonian (22). To this end one defines spin-isospin spherical harmonics [18]:

$$
\begin{aligned}
& \Xi_{G, M}^{( \pm, \pm)}=\left\langle\theta, \phi \mid G, M ; j=G \pm \frac{1}{2}, l=G \pm 1\right\rangle, \\
& \Xi_{G, M}^{( \pm, \mp)}=\left\langle\theta, \phi \mid G, M ; j=G \pm \frac{1}{2}, l=G\right\rangle,
\end{aligned}
$$

where $G, M, j$ and $l$ denote the eigenvalues of the grand-spin and its third component and of the total momentum and the angular momentum respectively. The soliton Dirac equation splits naturally into two parity $\mathcal{P}=\Pi(-)^{G}$ sectors. Natural parity $\Pi=(+)$ spinors are given by:

$$
\Psi_{G, M}^{+}(x)=\left(\begin{array}{c}
i A_{G}^{+}(r) \Xi_{G, M}^{(+,-)}+i C_{G}^{+}(r) \Xi_{G, M}^{(-,+)} \\
B_{G}^{+}(r) \Xi_{G, M}^{(+,+)}+D_{G}^{+}(r) \Xi_{G, M}^{(-,-)}
\end{array}\right)
$$

whereas for $\Pi=(-)$ they take the following form:

$$
\Psi_{G, M}^{-}(x)=\left(\begin{array}{r}
-i B_{G}^{-}(r) \Xi_{G, M}^{(+,+)}-i D_{G}^{-}(r) \Xi_{G, M}^{(-,-)} \\
A_{G}^{-}(r) \Xi_{G, M}^{(+,-)}+C_{G}^{-}(r) \Xi_{G, M}^{(-,+)}
\end{array}\right) .
$$

Then, for $\Pi=(+)$, the Dirac equation is reduced to a system of 4 first order differential equations for the radial functions $A \ldots D$ :

$$
\begin{aligned}
\frac{d}{d r}\left[\begin{array}{l}
A \\
B \\
C \\
D
\end{array}\right] & =\left[\begin{array}{cccc}
G / r & m \cos P+E & 0 & 0 \\
m \cos P-E & -(G+2) / r & 0 & 0 \\
0 & 0 & -(G+1) / r & m \cos P+E \\
0 & 0 & m \cos P-E & (G-1) / r
\end{array}\right]\left[\begin{array}{l}
A \\
B \\
C \\
D
\end{array}\right] \\
& +\frac{m \sin P}{2 G+1}\left[\begin{array}{cccc}
-1 & 0 & b_{G} & 0 \\
0 & 1 & 0 & -b_{G} \\
b_{G} & 0 & 1 & 0 \\
0 & -b_{G} & 0 & -1
\end{array}\right]\left[\begin{array}{l}
A \\
B \\
C \\
D
\end{array}\right],
\end{aligned}
$$

where $b_{G}=2 \sqrt{G(G+1)}$. Grand spin subscripts $G, M$ and parity superscript "+" have been suppressed. For $\Pi=(-)$ one should change the sign of $m$ in (6). For any soliton shape one can choose two different boundary conditions in the origin: 1) $A \sim r^{K}, \quad B \sim r^{K+1}$ and $C=D=0$ or 2) $A=B=0$ and $C \sim r^{K}, \quad D \sim r^{K-1}$. Therefore the solutions are labelled by a multiindex $n=(n, G, M, \Pi, i)$, where $n$ stands 
also for a radial quantum number and $i$ corresponds to the initial conditions discussed above.

In order to quantize the model one performs collective quantization in terms of the rotation matrix $A(t): U^{\gamma_{5}}(x)=A(t) U_{0}^{\gamma_{5}}(\vec{x}) A^{\dagger}(t)$, where $A^{\dagger} d A / d t=i / 2 \sum_{a=1}^{3} \tau_{a} \Omega_{a}$ [4]. As a result one gets a collective hamiltonian:

$$
\mathcal{H}_{\text {coll }}=M_{\text {sol }}+\frac{\overrightarrow{J^{2}}}{2 I}
$$

Here $M_{\text {sol }}$ is the soliton mass and $I$ the soliton moment of inertia. Spin operators $\vec{J}$ are related to the angular velocities by the quantization prescription: $I \vec{\Omega} \rightarrow \vec{J}$. The eigenstates of (7) have been successfully interpreted as baryons. Their wave functions are given in terms of $D^{(1 / 2)}(A)$ Wigner matrices and the moment of inertia $I$ is given as the sum over the eigenstates of the intrinsic Dirac hamiltonian (2) (in contrast to the collective hamiltonian (7)). Only the valence part of $I$ is here of interest:

$$
I_{\mathrm{val}}=\frac{N_{c}}{6} \sum_{n \neq \mathrm{val}} \frac{\langle\mathrm{val}|\vec{\tau}| n\rangle\langle n|\vec{\tau}| \mathrm{val}\rangle}{E_{n}-E_{\mathrm{val}}} .
$$

Let us note in passing that since $I \sim N_{\mathrm{c}}$ each power of $\Omega$ counts as one power of $1 / N_{\mathrm{c}}$.

Using the same path-integral formalism, which has been employed to derive $\mathcal{H}_{\text {coll }}$ one can derive a compact expression for any quark operator of interest. The space-like collective axial current operator can be expressed by the following functional trace in Euclidean space [13]:

$$
A_{j}^{a}=i \operatorname{tr}\left\{\Gamma_{j}^{b} D_{a b} \frac{1}{\partial_{t}+H\left(U_{0}^{\gamma_{5}}\right)+\frac{i}{2} \Omega_{c}^{\mathrm{E}} \lambda_{c}}\right\}
$$

with $\Gamma_{j}^{b}=\gamma_{4} \gamma_{j} \gamma_{5} \tau_{b}$ and $D_{a b}=1 / 2 \operatorname{Tr}\left(A^{\dagger} \tau_{a} A \tau_{b}\right)$. For a singlet axial current $\tau_{b}$ should be replaced by a unit matrix and one should also replace $D_{a b} \rightarrow 1_{a b}$ in eq.(9). It is implicitly understood that the sea part of eq.(9) is regularized and that the vacuum contribution, corresponding to $U_{0}^{\gamma_{5}} \rightarrow 1$ is subtracted.

Let us expand eq.(9) in $\Omega$, remembering that each power of $\Omega$ counts as $1 / N_{\mathrm{c}}$. In the zeroth order we get: 


$$
\left(A_{j}^{a}\right)^{(0)}=i \operatorname{tr}\left\{\Gamma_{j}^{b} D_{a b} \frac{1}{\partial_{t}+H}\right\} \equiv D_{a j} \alpha .
$$

Note that at this level the singlet axial current is equal to 0 in agreement with the Skyrme model result [5].

The next term in $\Omega$ (or in $1 / N_{\mathrm{c}}$ ) is of great importance. Expanding in $\Omega$ we get:

$$
\left(A_{j}^{a}\right)^{(1)}=\frac{1}{2} \operatorname{tr}\left\{\Gamma_{j}^{b} D_{a b} \frac{1}{\partial_{t}+H} \Omega_{c}^{\mathrm{E}} \lambda_{c} \frac{1}{\partial_{t}+H}\right\} .
$$

There are two subtleties connected with eq.(11): first it should be remembered that because of the collective quantization $\Omega_{c}$ is no longer a c-number but rather an operator $J_{c} / I$ which does not commute with $D_{a b}$. Second, the trace in eq.(11) should be understood as time-ordered [13 [15].

To this end let us consider the Euclidean propagator:

$$
\begin{aligned}
<x\left|\frac{1}{\partial_{t}+H}\right| y> & =\theta\left(t_{x}-t_{y}\right) \sum_{E_{n}>0} \Phi_{n}(\vec{x}) \Phi_{n}^{\dagger}(\vec{y}) \exp \left(-E_{n}\left(t_{x}-t_{y}\right)\right) \\
& -\theta\left(t_{y}-t_{x}\right) \sum_{E_{n}<0} \Phi_{n}(\vec{x}) \Phi_{n}^{\dagger}(\vec{y}) \exp \left(-E_{n}\left(t_{x}-t_{y}\right)\right) .
\end{aligned}
$$

If two such propagators are multiplied and time ordering in eq.(11) is assumed, then the correct expression for the baryon number one solutions reads (back in the Minkowskian space):

$$
\begin{array}{r}
\left(A_{j}^{a}\right)^{(1)=} \frac{i}{2 I} \sum_{\substack{E_{k}<\mu \\
E_{n}>\mu}} \frac{1}{E_{k}-E_{n}}\left\{<k\left|\Gamma_{j}^{b}\right| n><n\left|\tau_{c}\right| k>D_{a b} J_{c}\right. \\
\left.+<n\left|\Gamma_{j}^{b}\right| k><k\left|\tau_{c}\right| n>J_{c} D_{a b}\right\},
\end{array}
$$

where the chemical potential $E_{\mathrm{val}}<\mu<m$. Note that the order of $D_{a b}$ and $J_{c}$ is unambiguously dictated by the time ordering of the path-integral. One can always choose the basis $\mid \tilde{n}>$ (by a transformation of $\mid n>$ 's in each $G$ sector separately) such, that:

$$
<\tilde{n}\left|\Gamma_{j}^{b}\right| \tilde{k}>=<\tilde{k}\left|\Gamma_{j}^{b}\right| \tilde{n}>, \quad<\tilde{n}\left|\tau_{c}\right| \tilde{k}>=-<\tilde{k}\left|\tau_{c}\right| \tilde{n}>
$$


If at this point one does not pay attention to the order of $D_{a b}$ and $J_{c}$ in eq.(13), one can easily get a zero answer for $\left(A_{j}^{a}\right)^{(1)}$ in view of the symmetry properties of eq.(14). However, keeping the right order of the collective operators and making use of the property $\left[J_{a}, D_{j b}\right]=i \epsilon_{a b c} D_{j c}$, one finally arrives at the following expressions for the collective axial constants operators:

$$
g_{\mathrm{A}}^{(3)}=-\left(\alpha+\frac{\beta}{I}\right) \quad D_{33}, \quad g_{\mathrm{A}}^{(0)}=\frac{\gamma}{I} J_{3} .
$$

Note that the $1 / N_{\mathrm{c}}$ correction corresponding to $\beta / I$ has the same group-theoretical factor $D_{33}$ as the leading term in accordance with the general theorem of Ref. [19] The valence part of $\alpha$ entering eq.(15) and following from eq.(10) is given by:

$$
\alpha_{\mathrm{val}}=\frac{N_{c}}{3}\langle\text { val }|\vec{\sigma} \vec{\tau}| \operatorname{val}\rangle
$$

Furthermore from eq.(13):

$$
\begin{aligned}
\beta_{\mathrm{val}} & =-\frac{N_{c}}{6} i \epsilon_{i j k} \sum_{n \neq \mathrm{val}} \frac{\left\langle\mathrm{val}\left|\sigma_{i} \tau_{j}\right| n\right\rangle\left\langle n\left|\tau_{k}\right| \mathrm{val}\right\rangle}{E_{n}-E_{\mathrm{val}}} \operatorname{sign} E_{n} \\
\gamma_{\mathrm{val}} & =-\frac{N_{c}}{6} \sum_{n \neq \mathrm{val}} \frac{\langle\mathrm{val}|\vec{\sigma}| n\rangle\langle n|\vec{\tau}| \mathrm{val}\rangle}{E_{n}-E_{\mathrm{val}}}
\end{aligned}
$$

Now we will proceed to define the constituent quark limit more precisely. For the sake of simplicity we will use a somewhat unphysical profile function $P=\pi \theta\left(r_{0}-r\right)$ and then tune $r_{0} \rightarrow 0$. In this limit the energy of the valence level $E_{\text {val }} \rightarrow m$ and the sea contributions to all quantities vanish. Simultaneously we will take the nonrelativistic limit, i.e. we will systematically neglect small components of the spinors (4) and (5). As we shall illustrate later the results are not changed for a realistic smooth profile function.

For the $\theta$ profile the term proportional to $\sin P$ in eq.(ब) vanishes and the radial functions $C(r) \equiv D(r) \equiv 0$ for all $r$, for boundary conditions $i=1$. Similarly $A(r) \equiv$ $B(r) \equiv 0$ for $i=2$. Therefore for given energy $E_{n}, G$ and $M$ we have four independent solutions corresponding to two parities and two boundary conditions: 


$$
\begin{aligned}
& \langle x \mid n, G, M,+, 1\rangle=\left(\begin{array}{r}
i A_{n G}^{+} \Xi_{G, M}^{(+,-)} \\
B_{n G}^{+} \Xi_{G, M}^{(+,+)}
\end{array}\right),\langle x \mid n, G, M,+, 2\rangle=\left(\begin{array}{c}
i C_{n G}^{+} \Xi_{G, M}^{(-,+)} \\
D_{n G}^{+} \Xi_{G, M}^{(-,-)}
\end{array}\right), \\
& \langle x \mid n, G, M,-, 1\rangle=\left(\begin{array}{c}
-i B_{n G}^{-} \Xi_{G, M}^{(+,+)} \\
A_{n G}^{-} \Xi_{G, M}^{(+,-)}
\end{array}\right),\langle x \mid n, G, M,-, 2\rangle=\left(\begin{array}{c}
-i D_{n G}^{-} \Xi_{G, M}^{(-,-)} \\
C_{n G}^{-} \Xi_{G, M}^{(-,+)}
\end{array}\right) .
\end{aligned}
$$

For the valence level "val" corresponds to $(n=0, G=0, M=0, \Pi=+, i=1)$. Note that $\Xi_{0,0}^{(-, \pm)}$identically vanish, so for $G=0$ there is no solution corresponding to the second boundary conditions $i=2$.

The nonrelativistic limit of eq.(6) can be obtained by approximating $E \approx m$. Then, for $r>r_{0} A^{+}$and $C^{+}$become large components of natural parity spinors, whereas for unnatural parity large components are given by $B^{-}$and $D^{-}$. For $r<r_{0}$ the upper and lower components are interchanged, since the sign of the mass term in eq.(6) flips, once $r$ crosses $r_{0}$. Therefore, the nonrelativistic limit corresponds to keeping $B^{+}=D^{+} \approx 0$ for $r>r_{0}$ and $A^{-}=C^{-} \approx 0$ for $r<r_{0}$. The normalized solutions can be obtained if the system is confined in a box of radius $R$. Then the normalization condition for the valence level in the nonrelativistic limit reads:

$$
\int_{0}^{r_{0}} d r r^{2}\left|B_{00}^{+}\right|^{2}+\int_{r_{0}}^{R} d r r^{2}\left|A_{00}^{+}\right|^{2}=1
$$

Obviously, in the limit $r_{0} \rightarrow 0$ the first integral has to vanish and the normalization is given entirely by the second term in eq.(20). One can similarly normalize other solutions corresponding to the nonvalence orbits.

Now, in order to calculate constants $\alpha_{\text {val }}, \beta_{\text {val }}, \gamma_{\text {val }}$ and the valence part of the moment of inertia we have to know the matrix elements of intrinsic operators between the states of eq.(19). Constant $\alpha_{\text {val }}$ can be easily evaluated by means of the WignerEckart theorem:

$$
\alpha_{\mathrm{val}}=-\frac{N_{\mathrm{c}}}{3}\left(-\int_{0}^{r_{0}} d r r^{2}\left|B_{00}^{+}\right|^{2}+3 \int_{r_{0}}^{R} d r r^{2}\left|A_{00}^{+}\right|^{2}\right) .
$$

In the limit $r_{0} \rightarrow 0$ the first integral vanishes and $\alpha_{\mathrm{val}}=-N_{\mathrm{c}}$, due to the normalization 
condition (20). The proton-spin-up matrix element of the collective operator $D_{33}$ is equal to $-1 / 3$, so the leading term of $g_{\mathrm{A}}^{(3)}$ is equal to $N_{\mathrm{c}} / 3$.

In order to evaluate the next term in eq.(15) let us first observe that the matrix elements $\langle n|\vec{\tau}|$ val $\rangle$ do not vanish only for unnatural parity $\mathrm{G}=1$ state $n=\left(n, 1, M^{\prime},-, 2\right)$ - this can be again easily verified by the Wigner-Eckart theorem. Here $M^{\prime}=0, \pm 1$ depending on whether we take $\tau_{3}$ or $\tau_{ \pm}$respectively. Since the ratio $\beta_{\text {val }} / I_{\text {val }}$ is dominated by the lowest energy level which has the energy $E_{0} \approx m$ the sums in (18) and (8) reduce actually only to the one term. After calculating $i \epsilon_{i j k}\left\langle\operatorname{val}\left|\sigma_{i} \tau_{j}\right| n\right\rangle$, where again $n=\left(0,1, M^{\prime},-, 2\right)$, one gets finally:

$$
\begin{aligned}
\beta_{\mathrm{val}}=\frac{N_{\mathrm{c}}}{3} \lim _{E_{0} \rightarrow m} \frac{1}{E_{0}-E_{\mathrm{val}}} & \left(\int_{0}^{r_{0}} d r r^{2} B_{00}^{+} C_{01}^{-}-\int_{r_{0}}^{R} d r r^{2} A_{00}^{+} D_{01}^{-}\right) \\
\times & \left(\int_{0}^{r_{0}} d r r^{2} B_{00}^{+} C_{01}^{-}+3 \int_{r_{0}}^{R} d r r^{2} A_{00}^{+} D_{01}^{-}\right) .
\end{aligned}
$$

Similarly:

$$
I_{\mathrm{val}}=\frac{N_{\mathrm{c}}}{2} \lim _{E_{0} \rightarrow m} \frac{1}{E_{0}-E_{\mathrm{val}}}\left(\int_{0}^{r_{0}} d r r^{2} B_{00}^{+} C_{01}^{-}-\int_{r_{0}}^{R} d r r^{2} A_{00}^{+} D_{01}^{-}\right)^{2} .
$$

The constituent quark limit (CQL) consists in taking $r_{0} \rightarrow 0$ and $E_{\text {val }} \rightarrow m$ :

$$
\frac{\beta_{\mathrm{val}}}{I_{\mathrm{val}}} \stackrel{\mathrm{CQL}}{\longrightarrow}-2
$$

Proceeding in a similar way one gets:

$$
\begin{aligned}
& \gamma_{\mathrm{val}}=-\frac{N_{\mathrm{c}}}{3} \lim _{E_{0} \rightarrow m} \frac{1}{E_{0}-E_{\mathrm{val}}}\left(\int_{0}^{r_{0}} d r r^{2} B_{00}^{+} C_{01}^{-}-\int_{r_{0}}^{R} d r r^{2} A_{00}^{+} D_{01}^{-}\right) \\
& \times\left(\int_{0}^{r_{0}} d r r^{2} B_{00}^{+} C_{01}^{-}+3 \int_{r_{0}}^{R} d r r^{2} A_{00}^{+} D_{01}^{-}\right),
\end{aligned}
$$

so that:

$$
\frac{\gamma_{\text {val }}}{I_{\text {val }}} \stackrel{\mathrm{CQL}}{\longrightarrow} 2
$$


Altogether we find:

$$
g_{\mathrm{A}}^{(3)} \stackrel{\mathrm{CQL}}{\longrightarrow} \frac{N_{\mathrm{c}}+2}{3} \quad \text { and } \quad g_{\mathrm{A}}^{(0)} \stackrel{\mathrm{CQL}}{\longrightarrow} 1
$$

in perfect agreement with the CQM results. One can convince oneself by a direct numerical calculation, that this result holds for any realistic soliton profile, whose size $r_{0} \rightarrow 0$. This is illustrated in Fig.1, where the $g_{\mathrm{A}}^{(3)}$ dependence on $r_{0}$ is plotted for $P=2 \arctan \left(\left(r_{0} / r\right)^{2}\right)$.

Now, in order to investigate the large soliton limit, it is enough to observe, that in this limit $\alpha \simeq \beta$ 15] in the sense of radial dependence and both scale like $r_{0}^{2}$ [15], whereas $I$ scales like $r_{0}^{3}$ [20]. Therefore for $r_{0} \rightarrow \infty$ the new $\mathcal{O}(1)$ terms are suppressed and the results of the Skyrme model are parametrically reproduced. However for large but finite $r_{0}$, the correction exists and differs from the Skyrme model in contrast to the chiral bag calculations in Ref. [16]. In the case of $g_{\mathrm{A}}^{(0)}$ the large soliton limit of $\gamma$ goes as inverse powers of $r_{0}$ [21], so that it is vanishing and the Skyrme limit $g_{\mathrm{A}}^{(0)}=0$ is reproduced. So we have in fact a smooth interpolation between the CQM and Skyrme model.

Summarizing: the axial coupling constants $g_{\mathrm{A}}^{(3)}$ and $g_{\mathrm{A}}^{(0)}$ evaluated in the $\mathrm{Nambu}-$ Jona-Lasinio model in a small soliton limit agree with the Constituent Quark Model. Since the NJL model is a nontrivial dynamical model with valence quarks and polarized Dirac sea, and the CQM is based on purely kinematical assumptions concerning the symmetries of the wave functions, this agreement is by no means obvious. It is only possible because of the subtle cancellation of the overlap integrals of a priori very different operators. It is also important that the overlaps inside the soliton vanish with the soliton size approaching 0 . Moreover, if the $\mathcal{O}(1)$ corrections coming from the time-ordering of the collective operators were not taken into account, the agreement for $g_{\mathrm{A}}^{(3)}$ would never be possible. For it is hard to believe that other corrections, like meson loops for example, which require extra regularization and envisage completely different 
dynamics, might bring about the similar cancellation (although this statement requires obviously quantitative analysis). Therefore we consider the results of this paper as a

further confirmation of the correctness of the procedure presented in Refs. [13] [15], where the detailed derivation of the $\mathcal{O}(1)$ rotational corrections has been presented.

\section{ACKNOWLEDGMENTS}

The authors acknowledge the support of Alexander von Humboldt Foundation (AB, MP) and Department of Energy grant DE-FG02-88ER40388 (AB). 


\section{REFERENCES}

[1] G.Karl and J.E.Paton, Phys. Rev. D30, 238 (1984).

[2] A.Manohar and H.Georgi, Nucl.Phys. B234, 189 (1984).

[3] S. Peris, Phys. Lett. B268, 415 (1991).

S. Weinberg, Phys. Rev. Lett. 67, 3473 (1991).

[4] G. Adkins, C. Nappi, and E. Witten, Nucl. Phys. B228, 552 (1983).

[5] S. Brodsky, J. Ellis, and M. Karliner, Phys. Lett. B206, 309 (1988).

[6] Y. Nambu and G. Jona-Lasinio, Phys. Rev. 122, 345 (1961).

[7] D.Diakonov, V.Petrov, and P.Pobylitsa, Nucl. Phys. B306, 809 (1988).

[8] H.Reinhardt and R.Wuensch, Phys. Lett. B215, 577 (1988).

[9] D.Diakonov, V.Petrov, and M.Praszałowicz, Nucl. Phys. B323, 53 (1989).

[10] Th.Meissner, F.Gruemmer, and K.Goeke, Phys. Lett. B227, 296 (1989).

[11] M. Wakamatsu and H. Yoshiki, Nucl. Phys. A524, 561 (1991).

[12] M. Wakamatsu and T. Watabe, Phys. Lett. B312, 184 (1993).

[13] A.Blotz, M. Praszałowicz, and K. Goeke, Phys. Lett. B317, 195 (1993).

[14] C. Christov et al., Phys. Lett. B325, 467 (1994).

[15] A.Blotz, M. Praszałowicz, and K. Goeke, RUB report No. TPII-41/93, 1993.

[16] A. Hosaka and H. Toki, Phys. Lett. B322, 1 (1993).

[17] J. Schechter and H. Weigel, Resolving Ordering Ambiguities in the Collective Quantization by Particle Conjugation Constraints, [hep-ph/9410321], 1994.

[18] S.Kahana and G.Ripka, Nucl. Phys. A429, 962 (1984).

[19] R. Dashen and A. Manohar, Phys. Lett. B315, 438 (1993).

[20] A.Blotz et al., Nucl. Phys. A555, 765 (1993).

[21] A.Blotz, M. Polyakov, and K. Goeke, Phys. Lett. B302, 151 (1993). 


\section{FIGURES}

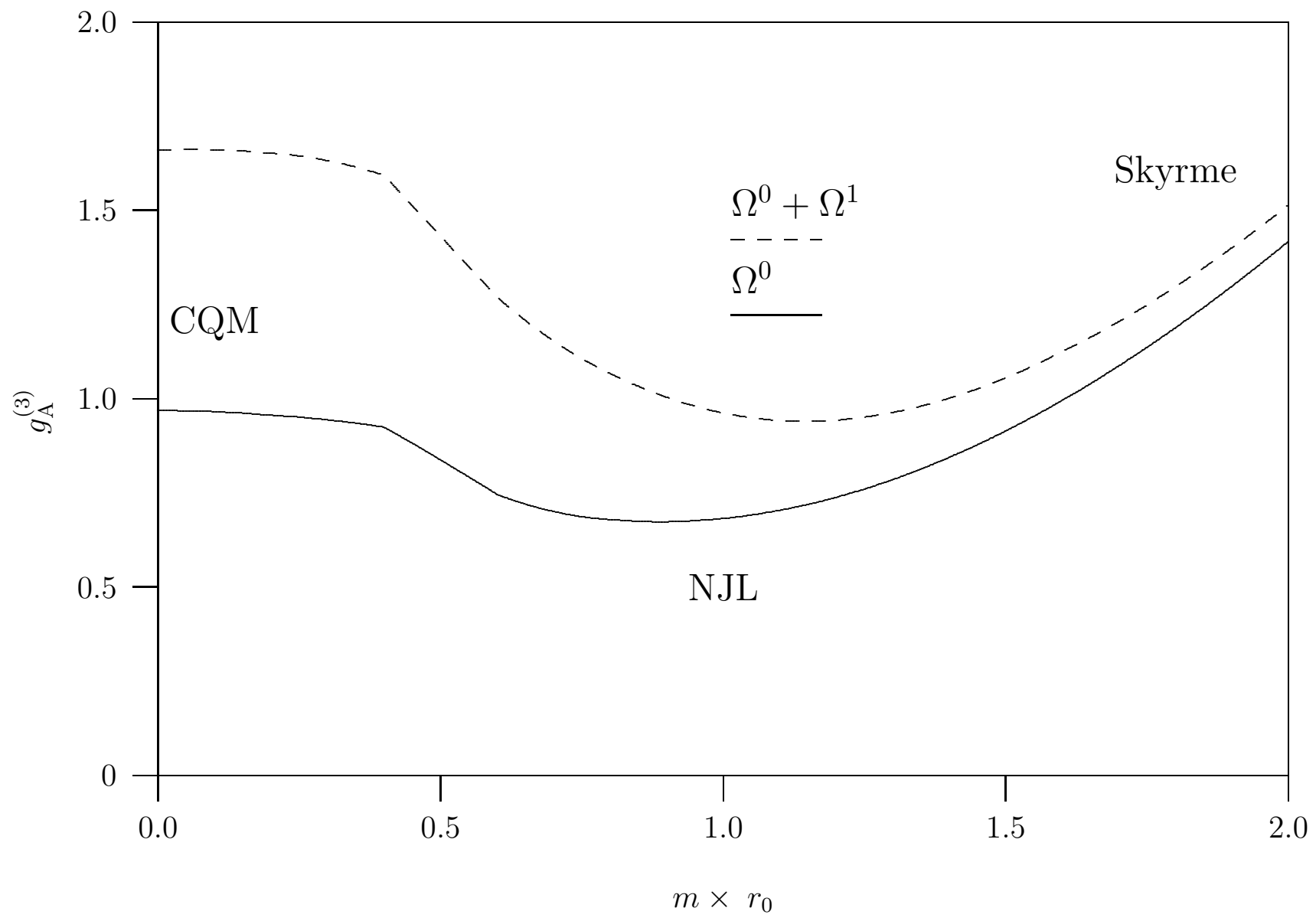

FIG. 1. The axial vector coupling constant $g_{\mathrm{A}}^{(3)}$ in dependence of the size $r_{0}$ of the chiral field for the lowest order $\Omega^{0}$ and next to leading order $\Omega^{0}+\Omega^{1}$ correction. The constituent quark mass is $m=370 \mathrm{MeV}$. 